\section{B CELLS}

\section{Staying power}

Adhesion molecules have an important role in the localization of marginal-zone (MZ) $B$ cells, according to a study published in the 19-July issue of Science. Lu and Cyster show that the integrins $\alpha 4 \beta 1$ and $\alpha \mathrm{L} \beta 2$ (also known as leukocyte function-associated antigen 1, LFA1) are essential for keeping MZ B cells in their place.

$\mathrm{B}$ cells in the splenic $\mathrm{MZ}$ - a structure that filters particulate antigens from the blood are phenotypically and functionally distinct from $B$ cells in the nearby follicles. But, the mechanisms that are responsible for the localization and segregation of MZ B cells have not been determined.

In this study, the expression of integrins on $\mathrm{MZ}$ and follicular B cells was compared; $\mathrm{MZ}$ $B$ cells were found to express higher levels of the integrins $\alpha 4 \beta 1$ and $\alpha L \beta 2$. Consistent with this, MZ B cells were shown to stick better than follicular $B$ cells to the $\alpha 4 \beta 1$ ligand vascular cell-adhesion molecule 1 (VCAM1) and the $\alpha \mathrm{L} \beta 2$ ligand intercellular cell-adhesion molecule 1 (ICAM1).
Immunohistochemical analysis of the spleen showed that ICAM1 and VCAM1 are indeed strongly expressed in the MZ.

To test the importance of $\alpha 4 \beta 1$-VCAM 1 and $\alpha \mathrm{L} \beta 2-\mathrm{ICAM} 1$ interactions for the localization of MZ B cells in vivo, mice were treated with blocking antibodies specific for $\alpha 4$ and $\alpha \mathrm{L}$. Within three hours, there was a complete exodus of $B$ cells from the MZ into the blood, but the B-cell follicles were unaffected, which confirms that these adhesive interactions have an essential role in the retention of $\mathrm{B}$ cells in the MZ.

$B$ cells migrate into the follicles in response to the chemokine CXCL13 - how do MZ $B$ cells resist this attraction? It seems that integrin interactions might be important, because follicular B cells were shown to migrate freely in response to CXCL13 on VCAM1-coated plates, whereas the migration of MZ B cells was inhibited.

Although MZ B cells do not recirculate, they migrate into the follicles in response to bacterial products and take antigens with them. Does integrin activity regulate this movement? Lipopolysaccharide (LPS)-treated MZ B cells had decreased adhesion to VCAM1 and were more responsive to CXCL13. When treated with LPS, the MZ B cells of Cxcl13 $3^{--}$

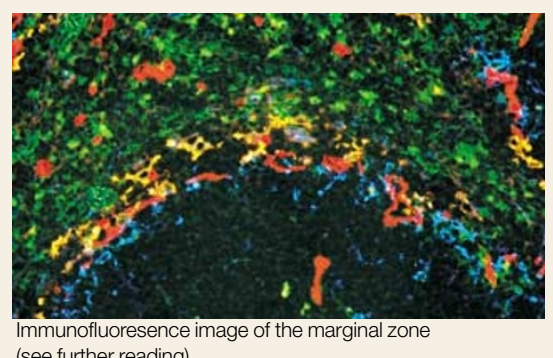

(see further reading)

mice relocated to the blood, rather than to the B-cell follicles, which indicates that downregulated integrin activity normally allows LPS-triggered MZ B cells to migrate to the follicles in response to CXCL13.

Importantly, these findings might explain the lack of MZ B cells in lymphotoxin $\alpha 1 \beta 2$ $(L t \alpha 1 \beta 2)^{---}, P y k 2^{-/-}$and $D o k 1^{--}$mice LT $\beta R$ signalling seems to be required for the expression of ICAM1 and VCAM1 in the MZ, and PYK2 and DOK1 are known to be signalling components downstream of integrin signalling.

(D) References and links Jennifer Bell original Research paper Lu, T. T. \& Cyster, J. G. Integrin-mediated long-term B-cell retention in the splenic marginal zone. Science 297, 409-412 (2002) FURTHER READING Martin, F. \& Kearney, J. F. Marginalzone B cells. Nature Rev. Immunol. 2, 323-335 (2002)

\section{VIRAL IMMUNITY}

\section{Combatting complement}

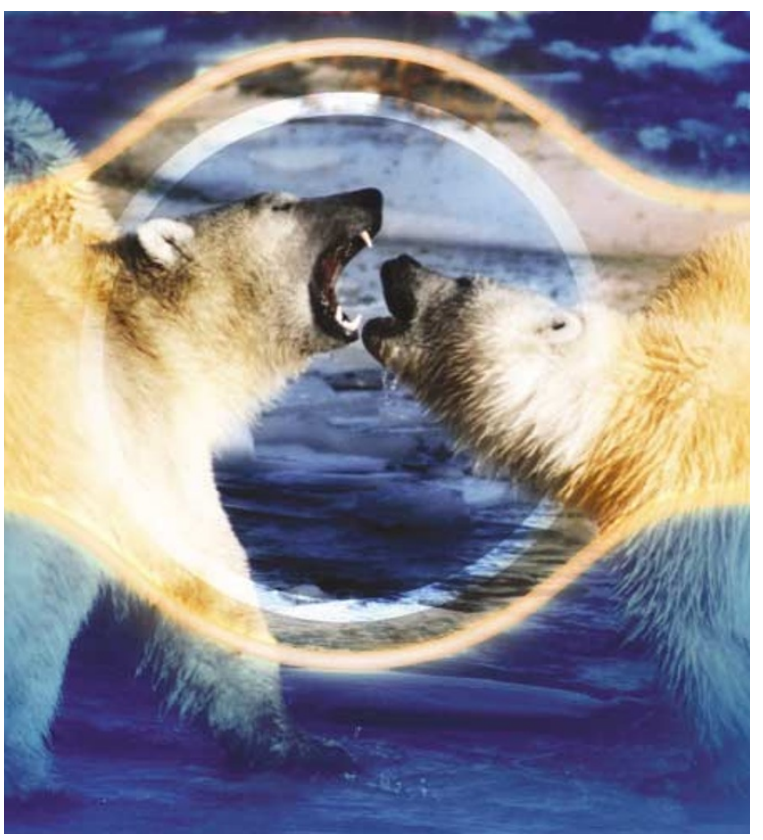

Work published in the Proceedings of the National Academy of Sciences by Rosengard and colleagues helps to explain one way in which variola virus, the causative agent of smallpox, successfully evades the human immune system, resulting in a mortality rate of $30-40 \%$ of infected individuals.

Variola virus specifically infects humans, and it is the most virulent member of the Orthopoxvirus family. By contrast, the closely related vaccinia virus causes no disease in humans. The difference in severity between these two Orthopoxvirus infections depends on host innate immune responses - including the complement system, which acts to destroy viruses and virus-infected cells - as well as viral immune-evasion mechanisms.

A better understanding of variola pathogenesis is required to develop a safer vaccine, but World Health Organization directives and ethical concerns preclude in vivo work on variola virus. As authentic variola proteins are unavailable, the authors used molecular-engineering techniques to characterize the complement-regulatory proteins (CRPs) of variola virus and vaccinia virus, and they compared their effectiveness in overcoming human complement activation.

The CRP that is encoded by smallpox smallpox inhibitor of complement enzyme (SPICE) - was generated by site-directed mutagenesis from its homologue, the vacciniavirus virulence factor vaccinia-virus complement-control protein (VCP). CRPs function as co-factors for the serine protease factor I to cleave $\mathrm{C} 3 \mathrm{~b}$ and $\mathrm{C} 4 \mathrm{~b}$ into inactive fragments. C3b-and C4b-degradation experiments showed that SPICE is $\sim 100$-fold more potent than VCP at inactivating human $\mathrm{C} 3 \mathrm{~b}$ and sixfold more potent at inactivating $\mathrm{C} 4 \mathrm{~b}$. These results indicate that SPICE might be a virulence factor for variola virus by protecting variola-infected cells from complement-mediated attack.

Next, the authors investigated the species preferences of these CRPs. SPICE preferentially inhibited human and baboon complement, whereas VCP preferentially inhibited dog and guinea-pig complement. These results show that variola virus exhibits human preference at a protein level, which potentially explains the preference of variola virus for a human host.

Variola proteins are, therefore, specifically able to evade the human immune response, and if smallpox were to re-emerge, SPICE might be a new therapeutic target.

(D) References and links

Jenny Buckland ORIGINAL RESEARCH PAPER Rosengard, A. M. et al. Variola virus immune evasion design: expression of a highly efficient inhibitor of human complement. Proc. Natl Acad. Sci. USA 99, 8808-8813 (2002)

FURTHER READING Smith, G. L. \& McFadden, G. Smallpox: anything to declare? Nature Rev. Immunol. 2, 521-527 (2002) 